\title{
Wirkung eines homöopathischen Kombinationsarzneimittels auf Tiergesundheit und Leistung bei Mastschweinen
}

\begin{abstract}
Title of the paper: Effect of a homoeopathic drug on animal health and performance in pigs

In the modern pig production the animals are still affected by sub-optimal condition. Economical reasons lead to high population densities in the stable, which can cause a higher susceptibility to diseases and misbehaviour. In the following trial growing pigs ( $70^{\text {th }}$ to $170^{\text {th }}$ days of living) were kept in four different groups with different space allowance (lowest possible space allowed by law vice versus higher space allowance). The trial group was treated with Engystol ${ }^{\circledR}$ via drinking water. Additionally to higher growing performance, the treated pigs had less lung and pleura affections as well as less withdrawing of lungs. In the groups of untreated animals tail biting did occur.

A treatment of growing pigs with Engystol ${ }^{\circledR}$ leads to higher growth performance and better meat quality. Although there will be no improved performance while the pigs are kept under optimal conditions, the susceptibility to negative influences like stress, diseases, poor environmental conditions will be reduced. A strong analysis of the given situation is clearly necessary. A treatment with Engystol ${ }^{\circledR}$ seems to be useful at specific points of interest like weaning, transport, movement.
\end{abstract}

Key Words: pig, homoeopathic treatment, health, meat quality, Engystol

\section{Zusammenfassung}

Auch in der modernen Schweineproduktion sind die Tiere Stressoren ausgesetzt. Ökonomische Gründe führen zu hohen Belegungsdichten, die Ursache für eine höhere Krankheitsanfälligkeit und Verhaltensanomalien sind. Im vorliegenden Versuch wurden wachsende Schweine (70. bis 170. Lebenstag) in vier verschiedenen Gruppen untersucht. Dabei wurden Tiere in niedriger und hoher Belegungsdichte (kleinstes rechtlich mögliches Platzangebot) gehalten. Die Versuchstiere erhielten Engystol $^{\circledR}$ über das Tränkwasser. Neben einer höheren Wachstumsleistung zeigten die behandelten Tiere auch eine geringere Anfälligkeit gegenüber Krankheiten, die sich bei den unbehandelten Gruppen vor allem in Lungen- und Brustfellveränderungen niederschlugen. Bei den unbehandelten Tieren trat Schwanzbeißen auf. Eine Behandlung von wachsenden Schweinen zur weiteren Ausschöpfung des Wachstumspotentials und Sicherung einer guten Fleischqualität kann durch die Gabe des Kombinationspräparates Engystol ${ }^{\circledR}$ erreicht werden. Dabei ist unter optimalen Haltungsbedingungen allerdings keine Leistungssteigerung zu erwarten, sondern der Nutzen beruht vielmehr auf einer geringeren Anfälligkeit gegenüber negativen Einflussfaktoren wie Stress, Krankheit, schlechte Umwelt. Eine genaue Einschätzung der Situation vor der Anwendung scheint unerlässlich, eine Anwendung nur zu bestimmten „Stresspunkten“ wie Absetzen, Umstallen u.ä. sinnvoll.

Schlüsselwörter: Schwein, homöopathisches Arzneimittel, Gesundheit, Fleischqualität, Engystol

\section{Einleitung}

Entscheidende Parameter für die letztendliche Qualität des Produktes „Schweinefleisch“ sind neben den eigentlichen Merkmalen der Fleischqualität auch Merkmale der Tiergesundheit und Wachstumsleistung der Tiere. Jede Form von Stress, jede Krankheit, auch die klinisch nicht sofort sichtbaren, beeinträchtigen die Leistung. Dabei werden die therapeutischen Möglichkeiten vor allem in der lebensmittelproduzie- 
renden Viehhaltung durch eine der Verbrauchererwartung gerecht werdenden Gesetzgebung immer engere Grenzen gesetzt. So wird der Schwerpunkt der Tiergesundheit mehr vom Bereich der Krankheitstherapie auf den Bereich der Vorbeugung gelegt. Auch die Begrenzungen der medikamentellen Pro- und Metaphylaxe machen die Suche nach neuen Behandlungswegen notwendig.

Eine der Behandlungsmöglichkeiten wird in der Anwendung der klassischen Homöopathie gesehen. Da die sehr aufwendigen Verfahren der klassischen Homöopathie für viele Nutztierhalter ökonomisch schwer tragbar sind und auf der anderen Seite viele Tierärzte keine Ausbildung auf diesem Gebiet haben, entsprechende Anwendungen aber dennoch vornehmen wollen, bietet der Markt Kombinationspräparate, deren Anwendung nach bewährten Indikationen erfolgt.

Mit der folgenden Untersuchung sollte herausgefunden werden, ob eine Anwendung des Kombinationspräparates Engystol $^{\circledR}$ über das Tränkwasser bei wachsenden Schweinen eine Verbesserung der Tiergesundheit, eine geringere Stressanfälligkeit und letztlich ein besseres Produkt für den Verbraucher liefert.

\section{Tiere}

\section{Material und Methoden}

Für den Versuch wurden Schweine der Deutschen Landrasse verwendet, die aus der Experimentalanlage Schwein des Forschungsinstitutes für die Biologie landwirtschaftlicher Nutztiere Dummerstorf stammen. Die Tiere sind am 70. Lebenstag aufgestallt und am 170. Lebenstag geschlachtet worden. Die Tiere stammen je Durchgang alle aus einer Abferkelphase. Um eine praxisnahe Situation zu gewährleisten, wurden die Gruppen nicht nach Verwandtschaftsverhältnis getrennt aufgestallt.

\section{Versuchsaufbau}

Der Versuch bestand aus zwei Durchgängen (Tab. 1). Im 1. Durchgang waren 34 Tiere in vier Gruppen eingeteilt - je eine Kontroll- und eine Versuchsgruppe mit zwei verschiedenen Belegungsdichten. Zwei Belegungsdichten wurden gewählt, um die Auswirkung der Belegungsdichte als Stressfaktor zu sehen.

In der Gruppe mit hoher Belegungsdichte waren die Tiere so dicht wie möglich im Rahmen der zulässigen Werte der Schweinehaltungsverordnung aufgestallt. Das unterschiedliche Platzangebot in den verschiedenen Alterstufen wurde durch unterschiedliche Tierzahlen pro Bucht realisiert. Das heißt, dass zu Versuchsbeginn sowohl in der Versuchs- als auch in der Kontrollgruppe 15 Tiere aufgestallt waren. Bei einem Durchschnittslebendgewicht der Gruppe von $50 \mathrm{~kg}$ wurden vier Tiere, bei einem Gewicht von $85 \mathrm{~kg}$ zwei weitere Tiere entfernt, so dass das Platzangebot den gesetzlichen Bestimmungen entsprach. Welche Tiere in die Auswertung aufgenommen wurden und welche als „Fülltiere“ fungierten, ist bei Versuchsbeginn durch zufällige Auswahl festgelegt worden.

Die Versuchs- und die Kontrollgruppe bei geringer Belegungsdichte bestanden konstant aus je acht Tieren, so dass sich mit zunehmendem Alter das Platzangebot zwar verringerte, aber immer deutlich über den gesetzlichen Vorgaben lag.

Die Auswertung des ersten Durchganges veranlasste uns, im 2. Durchgang auf die Gruppen mit geringer Belegungsdichte zu verzichten und zwei Versuchs- und zwei Kontrollgruppen mit je neun Versuchstieren entsprechend der maximalen Belegungs- 
dichte der gesetzlichen Vorgaben aufzustallen. Das Verfahren zur Platzangebotsregulierung entsprach demjenigen im ersten Versuch.

Tabelle 1

Versuchsaufbau, Tiergruppen und Behandlungsdauer (Trial-Setup, animal groups, duration of treatment)

\begin{tabular}{|c|c|c|c|}
\hline \multicolumn{4}{|c|}{ Durchgang 1 Versuchsgruppe (gleiche Anzahl der Tiere für Kontrollgruppe) } \\
\hline & Aufstallung & Behandlungsbeginn & Schlachtung \\
\hline Lebenstag & 70 & 100 & 170 \\
\hline Lebendgewicht & Tiere/Bucht & Versuchstiere & Fläche/Tier $\left(\mathrm{m}^{2}\right)$ \\
\hline \multicolumn{4}{|c|}{ Hohe Belegungsdichte } \\
\hline $30-50 \mathrm{~kg}$ & 15 & 9 & 0,39 \\
\hline $50-85 \mathrm{~kg}$ & 11 & 9 & 0,53 \\
\hline $85-110 \mathrm{~kg}$ & 9 & 9 & 0,65 \\
\hline \multicolumn{4}{|c|}{ Niedrige Belegungsdichte } \\
\hline $30-50 \mathrm{~kg}$ & 8 & 8 & 0,74 \\
\hline $50-85 \mathrm{~kg}$ & 8 & 8 & 0,74 \\
\hline $85-110 \mathrm{~kg}$ & 8 & 8 & 0,74 \\
\hline \multicolumn{4}{|c|}{ Durchgang 2 Versuchsgruppe (gleiche Anzahl der Tiere für Kontrollgruppe) } \\
\hline & Aufstallung & Behandlungsbeginn & Schlachtung \\
\hline Lebenstag & 70 & 70 & 170 \\
\hline Lebendgewicht & Tiere/Bucht & Versuchstiere & Fläche/Tier $\left(\mathrm{m}^{2}\right)$ \\
\hline \multicolumn{4}{|c|}{ Hohe Belegungsdichte } \\
\hline $30-50 \mathrm{~kg}$ & 30 & 18 & 0,39 \\
\hline $50-85 \mathrm{~kg}$ & 22 & 18 & 0,53 \\
\hline $85-110 \mathrm{~kg}$ & 18 & 18 & 0,65 \\
\hline
\end{tabular}

\section{Behandlung}

Tiere der Versuchsgruppen erhielten Engystol ${ }^{\circledR}$ über das Tränkwasser - je Tier $2 \mathrm{ml}$ an zwei Tagen pro Woche (Montag und Donnerstag). Tiere des ersten Durchgangs erhielten das Kombinationspräparat vom 100. bis zum 170. Lebenstag, Tiere im zweiten Durchgang vom 70. bis zum 170. Lebenstag. Engystol ${ }^{\circledR}$ ist ein Kombinationspräparat (Hersteller: Biologische Heilmittel Heel GmbH, Baden-Baden), das nach homöopathischen Verfahren hergestellt wird und verschiedene Potenzen von Sulfur und Vincetoxicum enthält.

\section{Merkmale}

Die Wägung aller Tiere erfolgte einmal wöchentlich. Blutproben von jedem Tier wurden vor Versuchsbeginn und vor der Schlachtung genommen, sowie im zweiten Durchgang eine weitere Blutprobe fünf Wochen nach Versuchsbeginn. Im ersten Durchgang wurden Speichelproben entnommen: eine Probe vor Versuchsbeginn, in der ersten Versuchswoche von Montag bis Freitag täglich einmal, bis zur letzten Versuchswoche einmal wöchentlich und in der letzten Versuchswoche von Montag bis Freitag täglich. Die Schlachtung der Tiere erfolgte im institutseigenen Schlachthaus. Dabei wurden die Schlachtkörper auf krankheitsbedingte Veränderungen untersucht und nach einem vorgegebenen Schema bewertet (Scoring). Dabei wurde die Unversehrtheit der Haut (Verletzungen), Veränderungen an Gliedmassen, Augen, Ohren, Brustfell, Bauchfell, Darmpaket, Leber, Lunge und Herz beurteilt und anhand von vorgefertigten Listen Punkte vergeben. Je stärker die Veränderung war, desto mehr Punkte wurden vergeben (Keine Veränderung $=0$ Punkte, max. Punktzahl je nach Organ 2 bis 6). Ausgewertet wurde die Punktzahl für die einzelnen Organe und die Gesamtpunktzahl. 
Zur Beurteilung der Schlachtkörper und der Fleischqualität wurden Schlachtkörperund Warmgewichte bestimmt. In Höhe der 13./14. Rippe wurden die Rückenspeckdicke, die Kotelettfläche (mittels Bildanalyse), der Muskelfleischanteil (FOM-Gerät, FSK-Technology, Søborg, Dänemark) und die Helligkeit/Farbe (Chromameter CR 200, Minolta AG, Langenhagen, Deutschland) gemessen. An der Anschnittfläche des M. longissimus dorsi und des M. semitendinosus wurden 45 Minuten und 24 Stunden nach der Schlachtung der pH-Wert (pH-Star, Fa. Matthäus, Pöttmes, Deutschland), die Leitfähigkeit (LF-Star, Fa. Matthäus, Pöttmes, Deutschland) und die Impedanz (Meat Check 150, Fa. Sigma electronic, Erfurt, Deutschland) bestimmt.

\section{Untersuchungsmethoden}

Sowohl die Untersuchung der Kortisolkonzentration im Speichel als auch im Plasma sollen ein Maß für die Reaktion der Tiere auf Stress sein. In allen Blutproben wurde die Kortisolplasmakonzentration mittels kommerziellen Enzym-Immunoessay Kits (DSL, Inc. USA) bestimmt. Die Sensitivität des Essays lag bei 0,1 $\mathrm{gg} / \mathrm{dl}$, der Intraessay-Koeffizient bei 5,9\%, der Interessay-Koeffizient bei 7,9\%.

Zur Beurteilung des Gesundheitsstatus werden das Proliferationsvermögen der Lymphozyten (nur 2. Durchgang), Eiweiß- (nur 2. Durchgang) und IgG-Gehalt des Plasmas zu Beginn und Ende des Versuches, sowie die Tiere selbst vor der Schlachtung und als Schlachtkörper untersucht (Tab. 1). Um die Konzentration des Immunoglobulins G zu bestimmen, wurde ein indirekter kompetetiver ELISA-Test verwendet, der von MORROW-TESCH et al. (1994) beschrieben wurde und der auf der Verwendung von Kaninchen-anti-Schwein IgG Antikörpern (Sigma Chemical) basiert. Der IntraessayKoeffizient lag bei 4,1\%, der Interessay-Koeffizient bei 7,7\%. Proben zur Bestimmung des Speichelkortisolgehaltes wurden durch Kauen der Schweine auf Wattebällchen gewonnen (ein bis zwei Minuten bis zur Durchtränkung des Wattebällchens). Die Wattebällchen wurden bis zum Zentrifugieren in speziellen Röhrchen auf Eis gelagert. Zur Gewinnung des Speichels aus den Wattebällchen wurden diese für 10 Minuten bei 2500 x g zentrifugiert. Gewöhnlich können so 0,5 bis 1,0 ml Speichel gewonnen werden, die bis zur Bestimmung der Kortisolkonzentration bei $-20^{\circ} \mathrm{C}$ gelagert wurden. Die Bestimmung erfolgte mittels direktem Flüssigphase-Enzym-immunoessay (DRG International, Inc., USA) in $100 \mu$ l Doppelproben entsprechend der Herstellervorgaben. Die Kreuzreaktionen waren 60\% für Prednisolon, 29\% für Kortikosteron, 3\% für Kortison und 1\% für 11-deoxykortisol. Die Sensitivität des Essays lag bei 0,1 ng/dl, der Intraessay-Koeffizient bei 4,3\%, der Interessay-Koeffizient bei 5,8\%. Die mitogeninduzierte Lymphozytenproliferation in vitro gilt als Maß für die Kapazität der zellvermittelten Immunität. Die Bestimmung der Zellproliferation erfolgte mittels der bei TUCHSCHERER et al. (1998) ausführlich beschriebenen Methodik. Es wurden zwei Mitogene der Firma Sigma eingesetzt: das vorrangig T-Zellen stimulierende Concanavalin A (25 $\mu \mathrm{g} / \mathrm{ml}$, ConA) sowie das B-Zellen stimulierende Mitogen Lipopolysaccharid $(100 \mu \mathrm{g} / \mathrm{ml}$, LPS). Ein dimensionsloser Proliferationsindex ergibt sich aus dem Verhältnis der jeweils mitogenstimulierten Zellen zu den Kontrollzellen ohne Mitogenzusatz.

Für die statistische Auswertung wurde das SAS System für Windows Version 8 (SAS Institute, 1999) verwendet. Die LSMeans wurden mit der PDIFF Methode verglichen. 
Als Faktoren wurden die Belegungsdichte (nur im ersten Durchgang) sowie die Behandlung (in beiden Durchgängen) gewählt.

\section{Ergebnisse \\ Gewichte und Zunahmen}

Die Tiere der Versuchsgruppen aus beiden Durchgängen zeigen höhere Lebendmassen als die unbehandelten Tiere. Sichtbar wird der Unterschied ab der 4. Versuchswoche (LM4), signifikant wird der Unterschied ab der 8. Versuchswoche (Abb. 1). Die wöchentlichen Zunahmen in der 8., 9. und 11. Woche sind bei den Kontrolltieren signifikant niedriger als bei den behandelten Tieren. Mögliche Ursache dafür ist das Auftreten von Schwanzbeißern in der Kontrollgruppe (5 Tiere waren betroffen) des ersten Durchgangs und die damit geringeren Zunahmen der Tiere. Schwanzbeißen gilt als Verhaltensanomalie, die vor allem bei gestressten Tieren auftritt. Die betroffenen Tiere erholen sich zwar, erreichen aber nie wieder den Leistungsstand der behandelten Gruppe. Bei getrennter Analyse der beiden Durchgänge zeigt der erste Durchgang die bessere Ausschöpfung des Wachstumspotenzials in der behandelten Gruppe deutlicher als der zweite Durchgang. Dennoch liegen die Lebendmassen der behandelten Tiere zu allen Zeitpunkten über denen der unbehandelten Tiere.

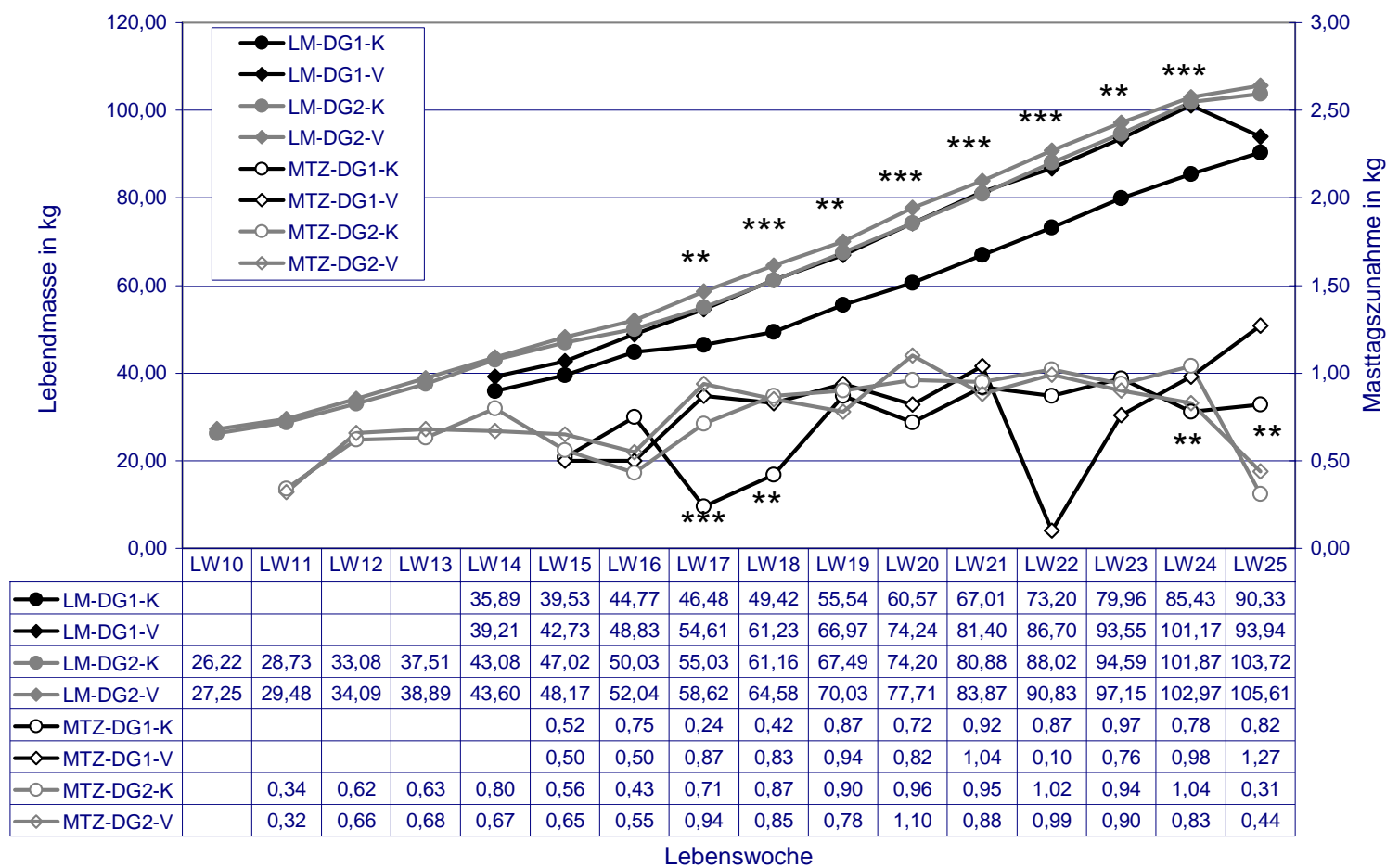

LSMean (K - Kontrollgruppe, V-Versuchsgruppe, DG1-1. Durchgang, DG2-2. Durchgang, LM-Lebendmasse, MTZ-Masttagszunahme, LW-Lebenswoche). Signifikanzen: * - p<0,1, ** - p<0,05, *** - p<0,01

Signifikante Unterschiede traten nur im 1. Durchgang auf, so dass sich die Signifikanzdarstellung darauf beschränkt.

Abb. 1: Lebendmassen (LM) und Masttagszunahme (MTZ) der Versuchs- und Kontrollgruppen mit hoher Belegungsdichte im ersten und zweiten Durchgang (Live weight (LM) and daily weight gain (MTZ) in the groups with high density in $1^{\text {st }}$ and $2^{\text {nd }}$ trial)

Zwischen den Tieren des ersten Durchganges, die in geringer Belegungsdichte gehalten wurden, können keine Unterschiede zwischen den Gruppen gefunden werden. 


\section{Kortisolkonzentrationen}

Wie in Abbildung 2 darstellt, sind zu Beginn der Untersuchung keine Unterschiede zwischen den Gruppen zu finden (es sind nur die dicht belegten Gruppen abgebildet, da in der gering belegten Gruppe keine Unterschiede auftreten). Signifikante Unterschiede treten zum Versuchsende auf. Die Tiere der behandelten Gruppe zeigen deutlich höhere Kortisolkonzentrationen als die unbehandelten Tiere. Eine getrennt Darstellung der beiden Durchgänge zeigt, dass es im ersten Durchgang zu einem Abfall der Konzentration kommt, bei den Kontrolltieren wesentlich deutlicher als bei den behandelten Tieren, während im 2. Durchgang ein Anstieg zu erkennen ist, der bei den behandelten Tieren deutlicher, wenn auch nicht signifikant, ausgeprägt ist.

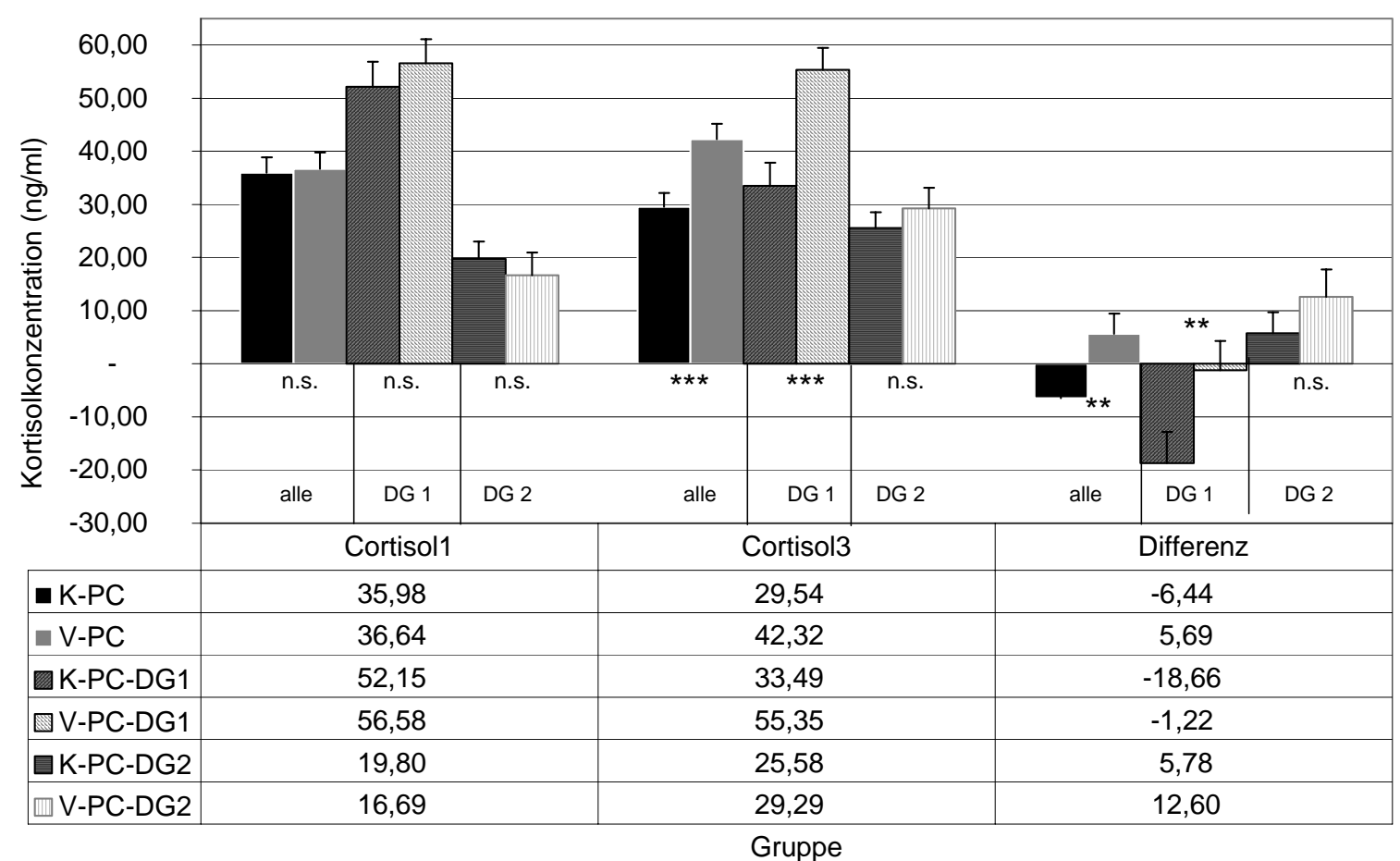

LSMean, (K - Kontrollgruppe, V-Versuchsgruppe, DG1 - Durchgang 1, DG2 - Durchgang 2). Angaben in ng/ml, Fehlerbalken zeigt den Standardfehler,

Signifikanzen: n.s. - nicht signifikant, * $-\mathrm{p}<0,1, * *-\mathrm{p}<0,05, * * *-\mathrm{p}<0,01$

Abb. 2: Plasmakonzentration von Kortisol (PC) (Concentration of Cortisol in plasma (PC))

\section{Gesundheitsbeurteilung}

Die Tiere der Kontrollgruppen zeigen höhere Werte im IgG-Gehalt (Tab. 2), die jedoch im Wesentlichen aus dem ersten Durchgang resultieren und als Reaktion der Körperabwehr auf das Schwanzbeißen gesehen wird. Sowohl der Proliferationsindex ConA als auch der Proliferationsindex LPS ist vom absoluten Wert her bei den behandelten Tieren höher und fällt mit dem Versuchsverlauf ab, während er bei den unbehandelten Tieren steigt.

Bei der Beurteilung der Tiergesundheit an Merkmalen des Schlachtkörpers sind vor allem Befunde an Brustfell und Lungen interessant (Abb. 3).

Während bei den Tieren in den gering belegten Buchten keine Unterschiede auftreten und generell ein guter Gesundheitsstatus erreicht wird, zeigen vor allem die Tiere der unbehandelten dicht belegten Gruppen in beiden Durchgängen deutlich höhere Auf- 
fälligkeiten hinsichtlich des Brustfells und der Gesamtpunktzahl im Scoring. Es kommt zu häufigeren Verwürfen von gesamten oder Teilen des Geschlinges.

Tabelle 2

Blutparameter; es sind nur Tiere der dicht belegten Gruppen eingeschlossen (Parameters in blood samples; only animals of the high density groups are shown)

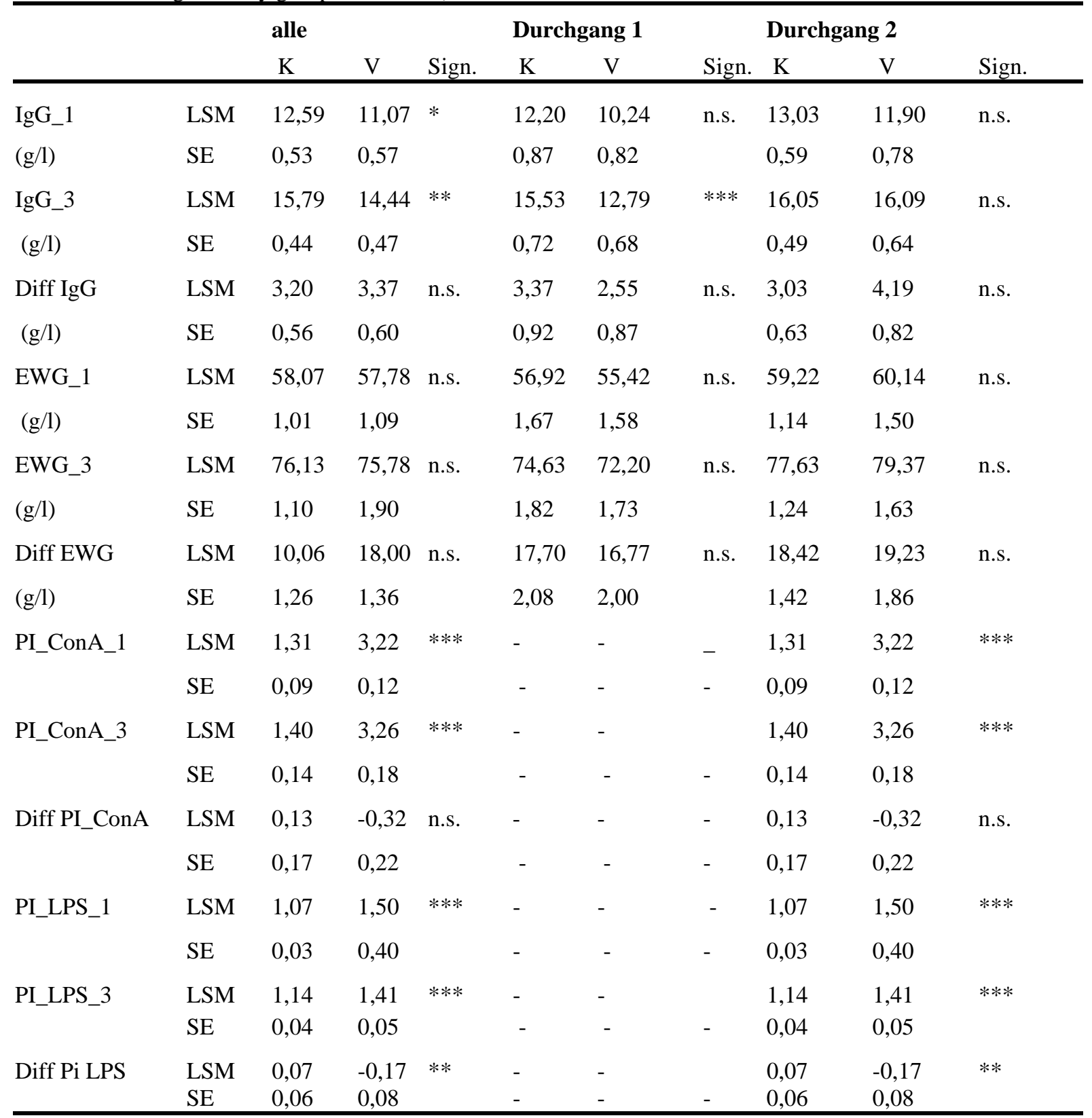

K - Kontrolle, V - Versuch, IgG - Immunglobulin G, EWG - Eiweißgehalt, PI_ConA - Concanavalin A, PI_LPS - Proliferationsindex Lipopolysacharidkomplex,

1 Zeitpunkt vor Versuchsbeginn, 3 - Versuchsende

Signifikanzen: n.s. - nicht signifikant, * $-\mathrm{p}<0,1, * *-\mathrm{p}<0,05, * * *-\mathrm{p}<0,01$ 


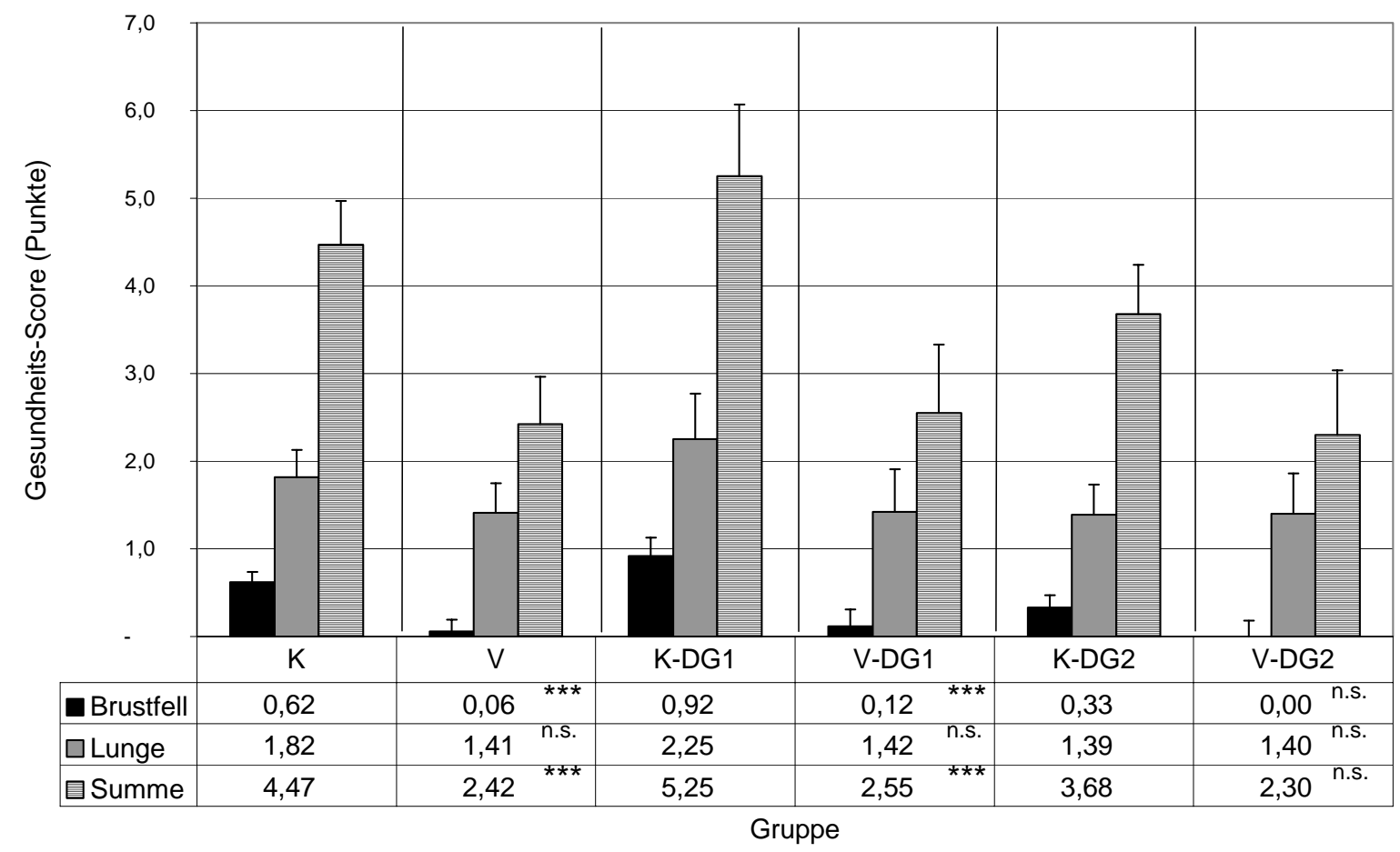

LSMean

K - Kontrollgruppe, V-Versuchsgruppe, DG1 - Durchgang 1, DG2 - Durchgang 2). Fehlerbalken zeigt den Standardfehler, Signifikanzen: n.s. - nicht signifikant, $*-\mathrm{p}<0,1, * *-\mathrm{p}<0,05, * * *-\mathrm{p}<0,01$

Abb. 3: Gesundheits-Score für ausgewählte Merkmale am Schlachtkörper (Health score for selected traits on carcass)

\section{Fleischqualität}

Zur Prüfung des Einflusses der Behandlung auf die Fleischqualität dient die Bestimmung verschiedener Merkmale der Schlachtkörper- und Fleischqualität. Während die Werte zwischen den Tieren der gering belegten Gruppen kaum Unterschiede aufweisen, zeigen sich bei den Tieren der dicht belegten Gruppen deutliche Unterschiede. Dabei erreichen die behandelten Tiere größere Gewichte und eine höhere Rückenspeckdicke. Unterschiede treten auch in der Kotelettfläche und im Muskelfleischanteil auf (Abb. 4).

Auch zwischen den Werten der Fleischfarbe gibt es signifikante Unterschiede. Die höhere Stressanfälligkeit der unbehandelten Tiere zeigt sich einer helleren Farbe (und damit schlechteren Qualität) des Fleisches $\left(L^{*}=48,10, S E=0,67\right)$ gegenüber den behandelten Tieren $\left(\mathrm{L}^{*}=45,54, \mathrm{SE}=0,71\right)$. 


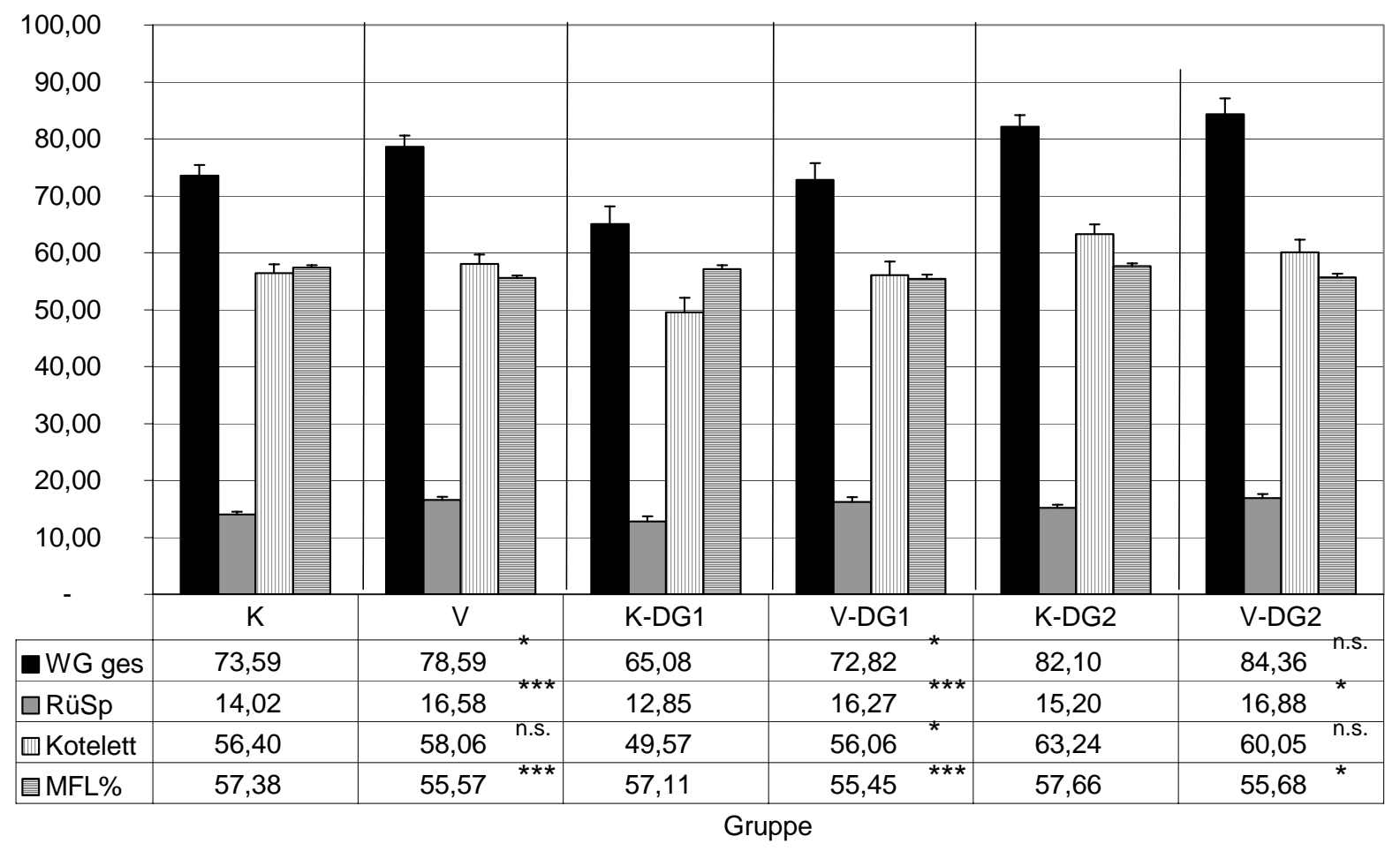

LSMean, WG ges - Warmgewicht gesamt (in kg), RüSp - Rückenspeckdicke (in mm), Kotelett (in $\mathrm{cm}^{2}$ ), MFL\% - Muskelfleischanteil (in $\%)$,

K - Kontrollgruppe, V-Versuchsgruppe, DG1 - Durchgang 1, DG2 - Durchgang 2). Fehlerbalken zeigt den Standardfehler,

Signifikanzen: n.s. - nicht signifikant, * ${ }^{*}<0,1, * *$ - $p<0,05, * * *-p<0,01$

Abb. 4: Qualitätsmerkmale am Schlachtkörper (Traits of Carcass quality

\section{Diskussion}

Der Einsatz von Engystol ${ }^{\circledR}$ bei anderen Tierarten hat bisher zwei wesentliche Wirkungsrichtungen aufgezeigt. Einmal tritt vor allem bei Ratten eine verminderte Stressreaktion auf (KIETZMANN, 1988). Zum anderen besteht eine positive Wirkung auf den Atmungstrakt, was bei verschiedenen anderen Tierarten gezeigt wird (Pferd KÜPPER, 1994; RIND - SCHARF, 1991, Mensch - MATUSIEWICZ, 1995). Beide Wirkungsziele sind für die Schweineproduktion bedeutsam. Da unter ökonomischem Druck die Belegungsdichten in den Schweineställen recht hoch sind (auch wenn die Gesetzgebung ein Mindestplatzangebot festlegt), zeigen vorliegende Untersuchungen, dass ein Ausschöpfen dieser rechtlichen Möglichkeiten bei den Schweinen zu Stressreaktionen (z. B. Schwanzbeißen) und dadurch zu verminderten Zunahmen führen kann. Bei ausreichend Platzangebot waren in vorliegenden Untersuchungen keine Unterschiede zwischen den Gruppen zu finden. Dass die Belegungsdichte einen sehr direkten Einfluss auf Lebendgewicht, tägliche Zunahme und Futterverwertung hat, wurde u.a. schon von HARPER et al. (1983) beschrieben. Verschiedene Einflussfaktoren auf das Auftreten von Schwanzbeißen werden in der Arbeit von MOINARD et al. (2003) dargestellt. Mehr Platzangebot und Verbesserung der Haltungsbedingungen durch Einstreu bringt die Tiere dazu, sich mehr mit der Umwelt zu befassen, sie zu erforschen und weniger aggressiv andere Schweine anzugehen oder inaktiv zu sein (BEATTIE et al., 2000). Nicht unerwartet zeigen auch neueste Untersuchungen in der Schweiz von CAGIENARD et al. (2005), dass Schweine einen positiven Gesundheitsstatus aufweisen, wenn sie unter guten Umweltbedingungen aufwachsen. 
Gerade im ersten Durchgang wird deutlich, dass eine hohe Belegungsdichte einen Stressfaktor darstellt und bei den Tieren Reaktionen auslösen kann (hier das Schwanzbeißen), die zu verminderter Leistung führen. Eine Möglichkeit, diesen eventuellen Stress durch eine hohe Belegungsdichte für die Tiere erträglicher zu machen und damit eine mögliche Leistungsdepression zu mindern, wird in der Gabe von Engystol $^{\circledR}$ gesehen. Auch wenn natürlich der direkte Nachweis, dass Engystol ${ }^{\circledR}$ ursächlich das Schwanzbeißen in der Versuchsgruppe des ersten Durchganges verhindert hat, nicht erbracht werden kann, ist doch die Tatsache, dass in beiden Durchgängen die Gewichte der behandelten Gruppe immer über denen der unbehandelten Gruppe liegen, ein deutliches Indiz für die bessere Ausnutzung des Wachstumspotenzials dieser Tiere.

Die Kortisolkonzentration wird als direkter Parameter zur Analyse von Stress-Situationen genutzt. Dabei kann die Kortisolkonzentration invasiv (Blut) oder nicht invasiv (Speichel) gemessen werden. Reaktionen der Kortisolkonzentration im Speichel auf Stresssituationen erfolgen erst bei sehr ausgeprägten Stressoren. DE GROOT et al. (2000) beschreiben eine verringerte circadiane Rhythmik des Speichelkortisolwertes bei Schweinen, die unter einfachen Bedingungen (kleine Buchten, kein Einstreu) gehalten werden gegenüber solchen aus größeren Buchten mit täglich frischer Einstreu. In der vorliegenden Untersuchung sind keine Unterschiede zwischen den Gruppen auszuweisen. Bei Betrachtung der Einzelwerte fallen große individuelle Unterschiede auf. Auch die Verläufe zwischen der Anfangs- und der Endmessung sind selbst innerhalb der einzelnen Gruppen sehr unterschiedlich. Den Haupteinfluss stellt der starke Abfall der Cortisolkonzentration in der Kontrollgruppe des ersten Durchganges dar. Die Ursachen dafür sind unbekannt.

Stressmindernde Wirkungen von Engystol ${ }^{\circledR}$, wie sie von KIETZMANN (1988) bei Ratten beschrieben werden, können mit den eigenen Versuchen nicht bestätigt werden. Ursachen dafür sind unterschiedliche Form und Dauer von Stresseinwirkung. Diese sind in den Versuchen nicht vergleichbar konstant im Gegensatz zu den Rattenversuchen von KIETZMANN (1988).

Um den Gesundheitsstatus beurteilen und einen Einfluß auf das Immunsystem überprüfen zu können, dient die Bestimmung von Proliferationsindices der Lymphozyten. Die wenig deutlichen Aussagen hinsichtlich der Proliferationsionsindices der Lymphozyten decken sich mit Beobachtungen von DE GROOT et al. (2000). Diese besagen, dass Immunfunktionen durch die Gestaltung der Haltungsumwelt wenig beeinflusst werden.

Bei Untersuchungen der Schlachtkörper werden deutliche Unterschiede zwischen den Gruppen sichtbar. Sie manifestieren sich vor allem am Atmungsapparat. Die geringere Anzahl an Lungen- und Brustfellveränderungen sowie Lungen-Verwürfen bei den behandelten Tieren zeigen, dass auch beim Schwein eine deutliche Wirkung von Engystol $^{\circledR}$ auf den Atmungsapparat erwartet werden kann.

Letzendlich führt die Behandlung der Schweine mit Engystol ${ }^{\circledR}$ zu einem durchschnittlich 5 kg höherem Warmgewicht, dickerem Rückenspeck und einer größeren Kotelettfläche und damit zu einem ökonomischen Vorteil.

Schweine, die unter weniger guten Umweltbedingungen wachsen (wenig Platz, kein Einstreu) haben weniger schmackhaftes Fleisch und einen höheren Kochverlust, als Tiere, denen mehr Platz und Einstreu zur Verfügung steht (BEATTIE et al., 2000). 
Schweine mit ausreichend Platzangebot weisen einen hohen $\mathrm{pH}-$ Wert in der Zeit von bis zu 4 Stunden post mortem auf, der durch die geringere Laktatkonzentration bedingt ist. Der End-pH-Wert ist allerdings am niedrigsten. Fleisch von konventionell gehaltenen Tieren zeigt einen höheren Dripverlust. LAMBOOIJ et al. (2004) schlussfolgern, dass die Umweltbedingungen unter denen die Schweine wachsen nur einen geringen Einfluss auf die Fleischqualität haben, wobei aber der größere Bewegungsspielraum bei geringer Belegungsdichte zu einem niedrigeren Dripverlust führt. Merkmale der Fleischqualität werden in den vorliegenden Versuchen nur wenig beeinflusst, weil die Unterschiede in der Umweltgestaltung zu gering sind.

\section{Schlussfolgerung}

Die Verwendung von Engystol ${ }^{\circledR}$ kann die Reaktion von Schweinen auf Stressoren und damit verbundene Leistungsdepressionen mindern. Dadurch werden auch Auswirkungen negativer Einflüsse, wie Erkrankungen, Verhaltensanomalien abgefedert und das Wohlbefinden der Tiere gefördert. Ökonomische Verluste der Tierhalter können verringert werden. Bei Tieren, die unter optimalen Bedingungen gehalten werden, sind keine deutlichen Wirkungen zu erwarten.

\section{Literatur}

BEATTIE, V.E.; O'CONNELL, N.E.; MOSS, B.W.:

Influence of environmental enrichment on the behaviour, performance and meat quality of domestic pigs. Livest. Prod. Sci. 65 (2000), 71-79

CAGIENARD, A.; REGULA, G.; DANUSER, J.:

The impact of different housing systems on health and welfare of grower and finisher pigs in Switzerland. Preventive Veterinary Medicine 68 (2005), 49-61

DE GROOT, J.; DE JONG, I.C.; PRELLE, I.T.; KOOLHAAS, J.M.: Immunity in barren and enriched housed pigs differing in baseline cortisol concentration. Physiology \& Behaviour 71 (2000), 217-223

HARPER, A.F.; KORNEGAY, E.T.:

The effects of restricted floor space allowance and virginiamycin supplementation on the feedlot performance of swine. Livest. Prod. Sci. 10 (1983), 397-409

KIETZMANN, M.:

Versuche mit Engystol $^{\circledR}$ (-Heel), 1. Vorversuch zur Dosisfindung. Biol. Tiermedizin 3 (1986), 26-33

KIETZMANN, M.:

Versuche mit Engystol ${ }^{\circledR}$ (-Heel), 2. Intermediäre Wirkung der Inhaltsstoffe. Biol. Tiermedizin 5 (1988), 38-50

KÜPPER, G.:

Atemwegserkrankungen beim Pferd - Prophylaxe und Therapie mit Engystol ad us. vet.. Biol. Tiermedizin 11 (1994), 19-31

LAMBOOIJ, E.; HULSEGGE, B.; KLONT, R.E.; WINKELMAN-GOEDHART, H.A.; REIMERT, H.G.M.; KRANEN, R.W.:

Effects of housing conditions of slaughter pigs on some post mortem muscle metabolites and pork quality characteristics. Meat Science 66 (2004), 855-862

MATUSIEWICZ, R.:

Wirksamkeit von Engystol N bei Bronchialasthma unter kortikoidabhängiger Therapie. Biol. Medizin 5 (1995), 242-246

MOINARD, C.; MENDL, M.; NICOL, C.J.; GREEN, L.E.:

A case control study of on-farm risk factors for tail biting in pigs. Applied Animal Behaviour Science 81 (2003), 333-355

MORROW-TESCH, J.L.; McGLONE, J.J.; SALAK-JOHNSON, J.L.:

SAS INSTITUTE,:

Heat and social stress effects on pig immune measures. J. Anim. Sci. 72 (1994), 2599-2609

SAS/STATR User’s Guide, Version 8. SAS Institute Inc, Cary, NC. (1999) 
SCHARF, K.G.:

Engystol ad us. vet. als Basismittel bei einem Ausbruch von Rindergrippe. Biol. Tiermedizin 8 (1991), 131-132

TUCHSCHERER, M.; PUPPE, B.; TUCHSCHERE, A.; KANITZ, E.:

Effects of social status after mixing on immune, metabolic, and endocrine responses in pigs. Physiol. Behav. 64 (1998), 356-360

Eingegangen: 04.08.2005

Akzeptiert: 17.05.2006

Anschriften der Verfasser

Dr. OLAF BELLMANN*, Prof. KLAUS ENDER

Forschungsbereich Muskelbiologie und Wachstum

Forschungsinstitut für die Biologie landwirtschaftlicher Nutztiere

W.-Stahl-Allee 2

18196 DUMMERSTORF

* Autor für Korrespondenz, E-Mail: bellmann@fbn-dummerstorf.de

Dr. ELLEN KANITZ

Dr. MARGRET TUCHSCHERER

Forschungsbereich Haltungsphysiologie

Forschungsinstitut für die Biologie landwirtschaftlicher Nutztiere

W.-Stahl-Allee 2

18196 DUMMERSTORF

Dr. ERICH REINHART

Veterinärabteilung

Biologische Heilmittel Heel GmbH

Dr.-Reckweg-Strasse 2-4

76532 BADEN-BADEN 